\title{
HIV incidence estimate combining HIV/AIDS surveillance, testing history information and HIV test to identify recent infections in Lazio, Italy
}

\author{
Alessia Mammone ${ }^{1 *}$, Patrizio Pezzotti ${ }^{2}$, Claudio Angeletti ${ }^{1}$, Nicoletta Orchi ${ }^{1}$, Angela Carboni ${ }^{2}$, Assunta Navarra ${ }^{1}$, \\ Maria R Sciarrone ${ }^{1}$, Catia Sias ${ }^{1}$, Vincenzo Puro ${ }^{1}$, Gabriella Guasticchi ${ }^{2}$, Giuseppe Ippolito ${ }^{1}$, Piero Borgia ${ }^{2}$ and \\ Enrico Girardi', for the SENDIH Study Group
}

\begin{abstract}
Background: The application of serological methods in HIV/AIDS routine surveillance systems to identify persons with recently acquired HIV infection has been proposed as a tool which may provide an accurate description of the current transmission patterns of HIV. Using the information about recent infection it is possible to estimate HIV incidence, according to the model proposed by Karon et al. in 2008, that accounts for the effect of testing practices on the number of persons detected as recently infected.

Methods: We used data from HIV/AIDS surveillance in the period 2004-2008 to identify newly diagnosed persons. These were classified with recent/non-recent infection on the basis of an avidity index result, or laboratory evidence of recently acquired infection (i.e., previous documented negative HIV test within 6 months; or presence of HIV RNA or p24 antigen with simultaneous negative/indeterminate HIV antibody test). Multiple imputation was used to impute missing information. The incidence estimate was obtained as the number of persons detected as recently infected divided by the estimated probability of detection. Estimates were stratified by calendar year, transmission category, gender and nationality.
\end{abstract}

Results: During the period considered 3,633 new HIV diagnoses were reported to the regional surveillance system. Applying the model, we estimated that in 2004-2008 there were 5,465 new infections ( $95 \% \mathrm{Cl}$ : 4,538-6,461); stratifying by transmission category, the estimated number of infections was 2,599 among heterosexual contacts, 2,208 among men-who-have-sex-with-men, and 763 among injecting-drug-users. In 2008 there were 952 (6251,229) new HIV infections (incidence of 19.9 per 100,000 person-years). In 2008, for men-who-have-sex-with-men (691 per 100,000 person-years) and injecting drug users (577 per 100,000 person-years) the incidence remained comparatively high with respect to the general population, although a decreasing pattern during 2004-2008 was observed for injecting-drug-users.

Conclusions: These estimates suggest that the transmission of HIV infection in Lazio remains frequent and menwho-have-sex-with men and injecting-drug-users are still greatly affected although the majority of new infections occurs among heterosexual individuals.

Keywords: HIV incidence, Test for recent infection, Testing history, Avidity index

\footnotetext{
* Correspondence: alessia.mammone@inmi.it

"Istituto Nazionale per le Malattie Infettive "Lazzaro Spallanzani", Rome, Italy

Full list of author information is available at the end of the article
} 


\section{Background}

Estimating HIV incidence is essential for monitoring the evolution of the epidemic and evaluating the effectiveness of prevention efforts. However, providing accurate estimates of HIV incidence is a complex task. Measuring the seroconversion rate in cohort studies or repeated serosurveys is expensive and at best reveal incidence in high-risk groups [1] while routine surveillance systems record new HIV diagnoses and not new infections [2].

The application of new laboratory techniques in HIV/ AIDS routine surveillance systems to identify persons with recently acquired HIV infection has been proposed as a tool which may provide an accurate description of the current transmission patterns of HIV. These techniques are based on serological tests, usually defined as tests for recent infection (TRI), which use different algorithms to discriminate recent infections from longstanding ones using a single serum sample [3-5]. Their development was based on the dynamics of the humoral immune response during the post-seroconversion phase of HIV infection, and relies on the modifications of early HIV-1 antibodies over time during the early phase of the infection [6-8]. TRI are likely able to identify HIV infections that occurred within 5-7 months before the test with an overall median sensitivity of $88.8 \%$ (range $42.3-100 \%$ ) and a median specificity of $86.8 \%$ (range 49.5-100\%) [9], and some developed countries have implemented surveillance with TRI [10-16] either at a regional or national level. However, identifying recent infections does not directly provide an estimate of the incidence of HIV infection because persons who have recently been infected can delay the testing and are not necessarily tested in the first months after infection. Karon et al. proposed a statistical model combining HIV/AIDS surveillance data with TRI and testing history that provided estimates of incidence of HIV infection in the US [16,17]. Another study following a similar approach provided incidence estimates for France [18].

In Lazio, a region located in central Italy including the metropolitan area of Rome (almost six million inhabitants), a combined surveillance of HIV and AIDS cases has been active since 1985, [19] and since 2004, a TRI is performed in a substantial percentage of new HIV diagnoses through a multicentre study [20]. The test used to detect recent infections is based on measuring the Avidity Index (AI) of the HIV-1 specific antibodies which show a low avidity for the antigen in the early phase of the infection [21-23]. It can be performed by an automated enzyme immunoassay for antibodies to HIV, it is not expensive and has been shown to identify recent HIV infections with good accuracy [22-25].

The objective of this study was to provide an estimate of the incidence of HIV infection in this Italian region by using data provided by the routine surveillance system and TRIs, using the method proposed by Karon et al. [17].

\section{Methods \\ The regional surveillance system of HIV and AIDS diagnosis of the Lazio region}

Although a national HIV surveillance system was not implemented in Italy until 2009, in Lazio region, a mandatory Regional Surveillance System of HIV infections based on the anonymous notification of every HIV diagnosis by public and private laboratories and blood banks- has been established since 1985 [19]. HIV diagnosis is defined as a positive result on two consecutive assays for HIV antibodies performed with commercially available immunoenzymatic tests and confirmed by a positive Western blot.

For each individual diagnosed with HIV, laboratories fill a form with some personal identifying data [i.e., gender, date (i.e., day, month and year) and municipality of birth (country if born outside Italy) but not surname and name]. Then the form is sent to the regional AIDS units where the test result is given to the patient, and other information (i.e., risk factors, date of last documented negative test when available) are collected during the post-test counselling. All information are finally collected and analysed at regional level.

In order to identify multiple tests of the same subject, a linkage procedure, based on gender, date and municipality of birth is routinely performed on the HIV diagnosis reports. This generates a file of newly diagnosed HIV infection which is periodically updated. It is of note that this procedure can identify different individuals with the same gender, date and municipality of birth. However, when we simulated the specificity of this combination of information, using the National AIDS registry (see below for description), that collects all the personal identifying data for more than 60,000 cases, we found around 1 duplicate every 2,000 AIDS cases (i.e., 0.5 per 1,000$)$. To ensure confidentiality, this file is protected by safety procedures and can only be accessed for the purpose of surveillance.

Since 1985, following the indications of the Italian AIDS registry, in Lazio Region AIDS diagnoses are reported mandatorily. The case definition refers to the European definition. Complete identifying personal data are given in the form used and are routinely linked to the HIV diagnoses using a linkage procedure based on gender, date and municipality of birth. The combination of the two surveillance systems is called the Regional Surveillance System of HIV and AIDS (RSS).

In this study we selected all newly diagnosed HIV infections identified in the period from 2004-2008. 


\section{The SENDIH study}

The SENDIH (Studio Epidemiologico Nuove Diagnosi Infezione HIV-1) is a multicentre study which started in 2004. Characteristics and methods of the study have been previously described [20]. Briefly, the study collects the following information on newly diagnosed adults with HIV infection from 13 regional counselling and testing sites of the Lazio region: demographic data (age, gender, nationality, transmission category), date of last HIV documented negative test, clinical and laboratory data at diagnosis (including the presence of AIDS defining conditions, diagnosis of primary HIV infection, HIVRNA measurement, and CD4 cell count), and, after the individual has provided written informed consent, behavioural data. The study was approved by the ethics committee at the L. Spallanzani National Institute of Infectious Diseases, and all enrolled individuals provided written informed consent.

\section{Linkage procedure between RSS and SENDIH}

Given that neither RSS nor SENDIH collect complete identifying personal data, each centre participating in the SENDIH study was asked to send a file in which there was a record for each diagnosis containing both of the anonymous codes used for the RSS and the SENDIH. The data were then combined using these files.

\section{Definitions of recent and non recent infection}

Only individuals enrolled in SENDIH study could have been classified as having recent or long-standing infection. Recent infection identification was based on three different criteria: 1) if an individual had a documented negative HIV antibody test performed within 6 months before HIV diagnosis; 2) if he/she had laboratory evidence of HIV seroconversion at the time of diagnosis [i. e., presence of HIV RNA or HIV p24 antigen with simultaneous negative/indeterminate HIV antibody testing (HIV-1/2 ELISA and Western Blot)]; 3) an AI < 0.80 . To save laboratory resources, when one of the first two criteria is met, the AI is not performed because the individual is considered as having $\mathrm{AI}<0.80$.

An AI of HIV antibodies test was performed only if a serum sample was available within 2 months after the initial diagnosis, if the patient had a CD4 count $\geq 20$ cells/ $\mu \mathrm{L}$ and was clinical-AIDS-free. Moreover, since early treatment was found to affect the evolution of HIV antibody avidity [23], in newly diagnosed individuals who started anti-retroviral therapy, AI was not performed.

The AI of antibodies is calculated by an automated anti-HIV enzyme immunoassay (EIA), the AxSYM HIV 1/2gO (Abbott Diagnostics Division, Delkenheim, Germany), according to a procedure already described by Selleri [23]. An AI lower than 0.80 was selected to define a recent infection because identified as threshold with the highest accuracy (area under the receiver operating curve: 0.958 ) corresponding to a sensitivity of 93.0\% and a specificity of $98.5 \%$ [26]; this threshold was found to be associated with a mean window period of 202 days (standard error: 18.4 days) [27].

Conversely, patients were classified as having nonrecent infection if they had an $\mathrm{AI} \geq 0.80$.

\section{Incidence estimate of HIV infection}

To estimate the regional incidence of HIV infection, we used the model proposed by Karon et al. [17] that accounts for the effect of testing practices on the number of persons detected as being recently infected.

According to this method, incidence is estimated as the number of persons detected as being recently infected divided by the estimated probability of being detected as recently infected during the period of interest. The incidence estimator is:

$$
\mathbf{I}=\mathbf{r} /\left(\mathbf{p}_{1} * \mathbf{p}_{2} * \mathbf{p}_{3}\right)
$$

where $r$ is the number of recent infections detected, $p_{1}$ represents the estimated probability of having a HIV test within one year after infection (in brief it represents the population testing behaviour), which is different for individuals having a previous negative HIV test (defined as repeat testers) and for individuals at their first HIV test (new testers); $\mathrm{p}_{2}$ is the percentage of persons with a newly reported HIV infection that had a TRI result; and $\mathrm{p}_{3}$ is the probability of being classified as recently infected, given a sample obtained at most one year after infection.

Incidence of HIV infection was firstly estimated using original data, assuming that the testing history data and the AI results are missing completely at random. However, in a logistic regression model, significant predictors $(\mathrm{p}<0.001)$ of missing testing history information were transmission category (three categories: MSM, IDU and heterosexual contacts) and type of diagnosis (four categories: AIDS diagnosis, (at HIV or within six months from HIV diagnosis), recent infection, non recent infection and HIV diagnosis AIDS-free with no criterion available for defining recent infection), while significant predictors $(\mathrm{p}<0.001)$ of missing AI results were transmission category, nationality (two categories: Italians and non-Italians), and history of previous HIV testing (two categories: Yes or No).

We then assumed that data on testing history and the AI results were missing at random and missing data were estimated by a two stage multiple imputation procedure.

First, history of previous HIV testing based on transmission category and type of diagnosis was imputed; at 
the same stage, conditionally on a previous imputed negative test, time delay between the two tests was imputed using a linear regression model based on transmission category and type of diagnosis as described above and 5 datasets were generated. Second, the AI results based on transmission category, nationality and history of previous HIV testing were imputed and 4 datasets were imputed from those obtained at the first stage (20 datasets in total).

For the original data and for each generated dataset with no missing information, for repeat testers, $\mathrm{p}_{1}{ }^{\mathrm{RT}}$ was estimated as the mean inverse inter-test time for repeat testers having a known last negative test date. For new testers, $\mathrm{p}_{1}{ }^{\mathrm{NT}}$ was estimated considering the proportion of HIV infections diagnosed at the AIDS stage and the distribution of the AIDS incubation periods, corresponding to a median incubation time of 8 years between infection and AIDS [28]. We used the US Centers for Disease Control and Prevention AIDS case definition [29] which is based on the presence of an AIDS defining illness or a CD4 count $<200$ cells $/ \mu \mathrm{L}$.

When estimating the incidence of HIV infection with original data, $\mathrm{p}_{2}$ was estimated separately for repeat and new testers; since $\mathrm{p}_{2}$ represents the probability that a person diagnosed with HIV had a TRI that could result in being classified as recent, $\mathrm{p}_{2}$ is estimated not considering persons with an initial diagnosis of AIDS or who develop AIDS within 6 months of their HIV diagnosis. When using the generated dataset with no missing information $\mathrm{p}_{2}$ is one.

For $\mathrm{p}_{3}$, a mean window period for an $\mathrm{AI}<0.80$ of 202 days was considered [27]. We assumed that also patients not tested with AI but identified as recently acquired infections with the other criteria have an $\mathrm{AI}<0.80$.

For each dataset, the total incidence estimate was then the sum of the estimated incidence for new testers,

$$
\mathrm{I}^{\mathrm{NT}}=\mathrm{r}^{\mathrm{NT}} /\left(\mathrm{p}_{1}{ }^{\mathrm{NT}} \mathrm{p}_{2}{ }^{\mathrm{NT}} \mathrm{p}_{3}\right)
$$

and the estimated incidence for repeat testers,

$$
\mathrm{I}^{\mathrm{RT}}=\mathrm{r}^{\mathrm{RT}} /\left(\mathrm{p}_{1}{ }^{\mathrm{RT}} \mathrm{p}_{2}{ }^{\mathrm{RT}} \mathrm{p}_{3}\right) .
$$

Variances and 95\% confidence intervals reported were calculated using the method recently proposed by Carnegie [30].

For the imputed datasets, the estimates reported are the mean of the 20 values obtained, while the lower and the upper bound of the $95 \% \mathrm{CI}$ were assumed to be respectively the minimum of the lower bound and the maximum of the upper bound, among the 20 estimates.

Estimates were stratified by calendar year, gender, transmission category and nationality (i.e., Italian, nonItalian).
To estimate incidence rates (overall or stratified by calendar year or gender), we used annual estimates of the population aged 15 years old or more residing in the region of Lazio from the National Bureau of Census http://www.demo.istat.it. The number of injecting drug users were obtained using the prevalence of heroin users in Italy from 2004-2008 estimated by the European Monitoring Centre for Drugs and Drug Addiction, corrected by the proportion of injecting subjects [31]. The estimated size of the non-national population living in Lazio, including documented and non-documented immigrants, was obtained from the Dossier Statistico Caritas/Migrantes [32]. The number of men who have sex with men was calculated as $3.1 \%$ of males aged 18-70, based on the results of a survey on sexual behaviours recently conducted in Italy [33].

Analyses were done with R software version 2.12 [34]. In particular package mi [35] was used for multiple imputation and package hivi [30] was used to compute 95\% confidence intervals of estimates.

\section{Results}

During the period 2004-2008, 3,633 new HIV diagnoses were reported to the RSS within the end of 2010 (Table 1). Among them, 2,170 (60\%) were diagnosed in SENDIH sites. Individuals diagnosed in SENDIH were more frequently men $(79 \%$ vs. $70 \%$ in the non-SENDIH), MSM (53\% vs. 26\%) and less frequently heterosexuals (38\% vs. $63 \%$ ); no difference was observed by age and for the percentage of IDU and foreigners. Of the 3,633 HIV diagnoses, 886 (24.4\%) were classified as having AIDS according to the CDC definition (of whom 69.4\% had a CD4 count $<200$ cells/ $\mu \mathrm{L}$ and no AIDS-defining illness) and a further 512 (14\%) were classified as longstanding infections based on AI results. Two hundred ninety $(8 \%)$ individuals were classified as recent

Table 1 New HIV diagnoses by type of diagnosis and information on a previous negative HIV test; Lazio, Italy, 2004-2008

\begin{tabular}{lccccc}
\hline & \multicolumn{5}{c}{ Ever Tested HIV Negative? } \\
\cline { 2 - 6 } & Yes & No & Unknown & Total \\
\hline $\begin{array}{l}\text { AIDS* within 6 months of HIV } \\
\text { diagnosis }\end{array}$ & 19 & 65 & 100 & 184 \\
AIDS* diagnosis at HIV diagnosis & 102 & 324 & 276 & 702 \\
HIV diagnosis, non-recent infection & 278 & 194 & 40 & 512 \\
HIV diagnosis, recent infection & 190 & 55 & 45 & 290 \\
HIV diagnosis, no test for recent & 201 & 177 & 1,567 & 1,945 \\
infection & & & & \\
\hline Total & $\mathbf{7 9 0}$ & $\mathbf{8 1 5}$ & $\mathbf{2 , 0 2 8}$ & $\mathbf{3 , 6 3 3}$ \\
\hline
\end{tabular}

*Diagnoses at the AIDS stage were considered as non-recent regardless of avidity index results. AIDS definition used: clinical AIDS or CD4 count $<200$ cells/ $\mu \mathrm{L}$ 
infections: in $52 \%$ of the cases classification was based on AI results, $30 \%$ had a documented negative HIV test in the previous 6 months, while the remaining 18\% had laboratory evidence of HIV seroconversion. One thousand-nine-hundred-forty-five individuals (53.5\% out of the total, 34\% out of those diagnosed in SENDIH sites) were AIDS-free at the time of diagnosis of HIV but no criterion to establish a recent infection was available. Information about the history of previous HIV testing was available for $44.2 \%$ of all cases: the proportion of recent infection was $32.2 \%$ and $8.6 \%$ among classifiable patients with and without a previous HIV test, respectively.

After the multiple imputation procedure, overall, during the period 2004-2008 we estimated 5,465 new infections in the region of Lazio (95\%CI: 4,538-6,461) with an average of 1,099 (23.8 per 100,000) estimated infections per year (Table 2).

The number of newly diagnosed cases per year was quite stable during the study period (an average of 723 new diagnoses per year, range 682-786) as it was the number of estimated identified recent infections, except for a peak observed in 2006 (Table 3). The estimated infections per year varied greatly, ranging from a minimum of $952(19.9$ per 100,000) in 2008 to a maximum of 1,223 (26.8) in 2006 (Table 3).

When data were analyzed according to transmission category (Table 4), the higher number of new diagnoses was observed among heterosexual contacts (48.6\% of the total diagnoses) followed by MSM (41.9\%). The highest estimated number of identified recent infections was among MSM (440 out of a total of 921 estimated as identified during the study period, 48\%) and in this group there was a lower proportion of AIDS cases. The highest number of new HIV infections was estimated to have occurred among heterosexual contacts (2,599, 47\% out of the total), followed by MSM (2,208, 40\%) and by IDU $(763,13 \%)$.

With respect to gender and place of birth (Table 5), men account for $76 \%$ of the new diagnoses and persons born in Italy for $67 \%$, respectively; the estimated percentage of new diagnoses classified as recent infections tended to be higher in men than in women and in those born in Italy compared to foreign born individuals. The number of new infections estimated for men was approximately three times the number estimated for women, and cases estimated for Italians were slightly more than twice of those estimated for non-Italians (Table 5).

Table 6 shows the estimated incidence rates in some sub-groups (i.e., MSM, IDU, and non-Italians) by calendar year. Compared to estimates reported for the general population, on average, these rates are 30 times higher in MSM, 40 times higher for IDU and 3 times higher for non-Italians. Time trends in incidence for MSM and non-Italians were similar to the overall trend, while a constant decreasing trend was observed for IDU.

\section{Discussion}

During its first decade, the HIV epidemic in Italy was predominantly characterized by infections linked to intravenous drug use followed by those occurring among MSM. In this study we show that during the third decade of the epidemic, the majority of new HIV infections in an Italian region can be estimated to occur among heterosexual individuals. The HIV epidemic however, continues to disproportionately affect MSM and IDU. The overall estimated incident rates estimated for the period from 2004-2008 are of the same order of magnitude of those estimated for the 1990s, when the

Table 2 Parameter estimates, incidence estimates and 95\% confidence intervals (based on both the original and imputed data), Lazio, Italy, 2004-2008

\begin{tabular}{|c|c|c|c|c|}
\hline & \multicolumn{2}{|c|}{ Original data } & \multicolumn{2}{|c|}{ Imputed data $^{\dagger}$} \\
\hline & Repeat testers & New testers & Repeat testers & New testers \\
\hline$r$ & 190 & 55 & 620 & 301 \\
\hline$p_{1}$ & 0.522 & 0.136 & 0.398 & 0.205 \\
\hline$p_{2}$ & 0.696 & 0.585 & 1 & 1 \\
\hline Incidence estimate & 946 & 1,250 & 2,818 & 2,647 \\
\hline$(95 \% \mathrm{Cl})$ & $(857-1,035)$ & $(966-1,534)$ & $(2,305-3,426)$ & $(1,739-3,593)$ \\
\hline Diagnoses & 790 & 815 & 1,646 & 1,987 \\
\hline Total incidence estimate $(95 \% \mathrm{Cl})$ & \multicolumn{2}{|c|}{$\begin{array}{c}4,968 \\
(4,356-5,441)\end{array}$} & \multicolumn{2}{|c|}{$\begin{array}{c}\mathbf{5 , 4 6 5} \\
(4,538-6,461)\end{array}$} \\
\hline Estimated incidence rate* & \multicolumn{2}{|c|}{21.1} & \multicolumn{2}{|c|}{23.8} \\
\hline
\end{tabular}

\footnotetext{
* per 100,000 person-years; estimates refer to the mean population (aged 15 or older) in the region of Lazio during the period $2004-2008$.

${ }^{+}$mean values from 20 imputations.

$P_{3}=202 / 365.25=0.553$.

$\mathrm{Cl}$, Confidence Interval.
} 
Table 3 Estimates of HIV incidence infections per calendar year, region of Lazio, Italy, 2004-2008

\begin{tabular}{cccccc}
\hline $\begin{array}{c}\text { Year of } \\
\text { diagnosis }\end{array}$ & $\begin{array}{c}\text { Newly diagnosed HIV } \\
\text { infections }\end{array}$ & $\begin{array}{c}\text { Identified Recent Infections } \\
\mathbf{N}(\%)^{*}\end{array}$ & $\begin{array}{c}\text { Estimate } \\
\text { infections }\end{array}$ & $\begin{array}{c}\mathbf{9 5 \%} \text { Confidence } \\
\text { Interval }\end{array}$ & $\begin{array}{c}\text { Estimated Incidence } \\
\text { rate }^{* *}\end{array}$ \\
\hline $\mathbf{2 0 0 4}$ & 760 & $184(24)$ & 1,144 & $843-1,516$ & 25.5 \\
$\mathbf{2 0 0 5}$ & 695 & $185(27)$ & 1,190 & $892-1,567$ & 26.2 \\
$\mathbf{2 0 0 6}$ & 786 & $216(27)$ & 1,223 & $925-1,636$ & 26.8 \\
$\mathbf{2 0 0 7}$ & 710 & $169(24)$ & 982 & $719-1,328$ & 20.8 \\
$\mathbf{2 0 0 8}$ & 682 & $167(24)$ & 952 & $625-1,229$ & 19.9 \\
Total & $\mathbf{3 , 6 3 3}$ & $\mathbf{9 2 1}$ & & & \\
\hline
\end{tabular}

* mean values from 20 imputations; $\%$ refers to the total of new diagnoses of each year

** per 100,000 person-years; estimates refer to the population (aged 15 or older) in the region of Lazio on January $1^{\text {st }}$ of each year

epidemic (according to the estimates based on back calculation methods) was stabilizing after an initial peak [36].

This is the first study that provides recent estimates of HIV incidence in an Italian region. The last estimates reported for the region of Lazio referred to 1992 with an estimate of around 2,000 new infections from a dynamic model [37]; in contrast, by using a back-calculation model, Bellocco et al. [38] estimated only 25003000 infections in Italy during 1994, corresponding to around 300-350 infections in Lazio region (based on the assumption that Lazio region has quite constantly accounted for $12 \%$ of the Italian AIDS cases each year and that this percentage holds also in the new HIV infections [39]). According to our estimates, in the period from 2004-2008 there was an average of around 1,099 infections per year. Although it is difficult to compare our results with previous estimates, the mean number of infections estimated for 2004-2008 remains surprisingly high.

There are no data available to provide a national estimate of HIV incidence by using this method. However, on the basis of our results it would be possible to provide a rough extrapolation of the expected number of new infections in the country, since HIV diagnosis and AIDS incidence in Lazio and the rest of Italy have similar temporal trends and similar characteristics in terms of gender, age and risk groups, [39] and given that there is free offer of treatment for infected persons in the whole country. In fact, considering that the region of Lazio has quite constantly accounted for $12 \%$ of the Italian AIDS cases each year, [39] and considering a mean estimated number of infections of around 1,099 cases each year in Lazio region, we could roughly estimate around 9,100 new infections each year in the entire country, which corresponds to an estimated incidence of around 15 per 100,000 .

Our incidence estimates generally varied by calendar year but there was no specific trend. While the peak was estimated in 2006, stratified analyses showed similar results in each sub-group except for injecting drug users for whom there was a clear decline of the estimated recent infections in the period from 2004-2008.

The statistical model used in this paper has been previously applied in the US and in France. Incidence estimates of 19.0 per 100,000 were obtained for the US [40] and of 17 per 100,000 for France, both in 2008 [18]; these estimates are similar to those obtained in the region of Lazio in the period 2004-2008.

Estimates of incidence rates by risk group provided important information about the HIV epidemic in our region. Regarding drug injection, the estimated number of new infections related to this behaviour had a decreasing trend during the period from 2004-2008, and new infections related to this behaviour represented approximately $14 \%$ of the overall cases estimated during the study period. However, in 2008 the estimated incidence rate was around 200 per 100,000, suggesting that

Table 4 Estimated new HIV infections, region of Lazio, Italy, 2004-2008, by transmission category (mean values from 20 imputations)

\begin{tabular}{|c|c|c|c|}
\hline & Heterosexual contacts & MSM & IDU \\
\hline Total newly diagnosed HIV infections & 1,765 & 1,523 & 345 \\
\hline Identified recent infection (\%)* & $371(21)$ & $440(29)$ & $110(32)$ \\
\hline Non-recent infection (\%)* & $920(52)$ & $765(50)$ & $141(41)$ \\
\hline AIDS case $(\%)^{*}$ & $474(27)$ & $318(21)$ & $94(27)$ \\
\hline Total incidence estimate $(95 \% \mathrm{CI})$ & $\begin{array}{c}2,599 \\
(1,930-3,386)\end{array}$ & $\begin{array}{c}\mathbf{2 , 2 0 8} \\
(1,641-2,689)\end{array}$ & $\begin{array}{c}763 \\
(476-1,059)\end{array}$ \\
\hline
\end{tabular}

*\% refers to the total number of diagnoses in each group

MSM, Men who have Sex with Men; IDU, Injecting Drug Users; Cl, Confidence Interval 
Table 5 Estimated new HIV infections, region of Lazio, Italy, 2004-2008, by gender and nationality (mean values from 20 imputations)

\begin{tabular}{ccccc}
\hline & MEN & WOMEN & ITALIANS & NON ITALIANS \\
\hline Total newly diagnosed HIV infections & $\mathbf{2 , 7 2 7}$ & $\mathbf{9 0 6}$ & $\mathbf{2 , 3 7 4}$ & $\mathbf{1 , 2 5 9}$ \\
\hline Identified recent infection (\%)* & $715(26)$ & $206(23)$ & $640(27)$ & 281 (22) \\
\hline Non-recent infection (\%)* & $1,319(49)$ & $507(56)$ & $1,139(48)$ & $687(55)$ \\
\hline AIDS case (\%) & $693(25)$ & $193(21)$ & $595(25)$ & $291(23)$ \\
\hline Total incidence estimate (95\% Cl) & $\mathbf{4 , 1 9 7}$ & $\mathbf{1 , 2 8 0}$ & $\mathbf{3 , 7 7 3}$ & $(1, \mathbf{7 0 3}$ \\
& $(3,373-4,978)$ & $(875-1,774)$ & $(2,886-4,617)$ & $(159-2,210)$ \\
\hline
\end{tabular}

*\% refers to the total number of diagnoses in each group

$\mathrm{Cl}$, Confidence Interval

IDU are still at high risk of infection. Also the MSM, who accounted for almost $40 \%$ of estimated infections, had an alarmingly high incidence rate that was around or above 500 per 100,000 in each year of the study. These results are consistent with those reported in France [18], where IDU and MSM were found to be disproportionately affected by the HIV epidemic, underscoring the urgent need to reinforce the prevention interventions that are targeted to these groups.

In this study we did not attempt to provide an estimate of the incidence rates in heterosexuals, given the wide heterogeneity of this group. Specific surveys are needed to provide an estimate of heterosexuals at high risk of HIV infection among whom incidence rates may be very high, as suggested by a recent study conducted in the US [41]. It is interesting to note that the higher number of infections was estimated among heterosexuals while the number of diagnosed HIV infections classified as recent infections was higher among MSM. This is likely due to the different testing behaviour of these two groups, $[42,43]$ and should be kept in mind when interpreting the results of observational studies that monitor the characteristics of recently acquired HIV infections. Furthermore, we should also consider that there is potential misclassification of MSM as heterosexual contact [44].

Although these results may be relevant to understanding recent trends of HIV epidemic in a low incidence country, several limitations should be considered.

The criteria used to define recent/non-recent infection are available only for persons diagnosed in SENDIH sites, and we assumed that incidence risk is the same for persons diagnosed in SENDIH and non-SENDIH sites. We assumed that individuals with missing information are missing at random and under this hypothesis the multiple imputation should have reduced potential bias in our estimates.

Table 6 Estimated new HIV infections and incidence, region of Lazio, Italy, 2004-2008, by transmission category and nationality

\begin{tabular}{ccccc}
\hline & Year & Estimated infections*(95\% Cl) & Estimated population size§ & Estimated incidence rate** \\
\hline \multirow{2}{*}{ MSM } & $\mathbf{2 0 0 4}$ & $466(267-714)$ & 55,853 & 834 \\
& $\mathbf{2 0 0 5}$ & $488(335-676)$ & 56,479 & 864 \\
& $\mathbf{2 0 0 6}$ & $506(292-714)$ & 56,641 & 893 \\
& $\mathbf{2 0 0 7}$ & $352(229-504)$ & 58,596 & 601 \\
\multirow{2}{*}{ IDU } & $\mathbf{2 0 0 8}$ & $408(238-561)$ & 59,043 & 691 \\
& $\mathbf{2 0 0 4}$ & $216(83-350)$ & 15,784 & 1,368 \\
& $\mathbf{2 0 0 5}$ & $195(59-400)$ & 11,752 & 1,659 \\
NON-ITALIANS & $\mathbf{2 0 0 6}$ & $158(64-298)$ & 12,666 & 843 \\
& $\mathbf{2 0 0 7}$ & $112(8-215)$ & 13,286 & 577 \\
& $\mathbf{2 0 0 8}$ & $74(6-181)$ & 12,818 & 73 \\
& $\mathbf{2 0 0 4}$ & $285(143-420)$ & 389,920 & 93 \\
\end{tabular}

* mean values from 20 imputations

§Population size estimates on January $1^{\text {st }}$ of each year; aged 18-70 years for MSM, 15-64 for IDU

**Per 100,000 person-years

MSM, Men who have Sex with Men; IDU, Injecting Drug Users; $\mathrm{Cl}$, Confidence Interval 
We combined three different criteria to define individuals with recent infection (i.e., an $\mathrm{AI}<0.80$, a previous documented negative test within 6 months, or laboratory evidence of recently acquired infection). In the model, we assumed that those identified as recent infection with the last two methods should have resulted with an $\mathrm{AI}<0.80$ if tested. Thus, it was reasonable to compute, also in this case, the incidence estimator as proposed by Karon. It is possible that some recent infections established with criteria other than AI, would have had an $\mathrm{AI} \geq 08$ and then classified as non-recent, but in this case we improved the sensitivity of the AI.

Other possible biases were described in detail in the article of Karon et al. [17] and these could also have biased our estimates. Among them, the AI test could have partly misclassified recent/non-recent infection because the accuracy of this test is not one, as assumed by the method. With regard to the reporting delays of HIV diagnoses, we can fairly exclude delays, since the period of interest was 2004-2008, the data were collected until the end of 2010 and the region considered is small.

The model assumes that the HIV test date is independent of the infection date for new testers, while for repeat testers it assumes that the risk of infection is constant between the last negative and first positive test date. Consequently the number of recent infections and thus incidence could be overestimated in people seeking HIV testing because of seroconversion symptoms or recent exposure. This issue could be addressed taking into account the reason for testing.

The history of testing and results of a TRI were not available for a significant proportion of new diagnoses. Preliminary analyses showed that results are strongly dependent on some parameters such as the window period of TRI used, the percentage of those with unknown testing history and the percentage of those without TRI result. We partly addressed this limit using multiple imputation that permits to correct the bias for missing at random information. Collecting information about HIV testing history and extending TRI to all new diagnoses reported to the regional surveillance system could improve the estimates limiting the effect of other source of bias.

Reliability of incidence estimates in specific subpopulations could be also affected by the accuracy of the subpopulation's size, such as IDU and MSM.

\section{Conclusion}

In conclusion, this study combined surveillance data with TRI to provide an estimate of the incidence of $\mathrm{HIV}$ infections in recent years in an Italian region. These estimates suggest that the transmission of HIV infection in the region of Lazio remains frequent and interventions for prevention should be considered. In order to improve HIV incidence estimates, public health departments should initiate programs to increase the percentage of TRIs performed and other regions should incorporate TRI results into their surveillance systems.

\section{Abbreviations}

Al: Avidity index; AIDS: Acquired immune deficiency syndrome; $\mathrm{Cl}$ : Confidence interval; HIV: Human immunodeficiency virus; IDUM: Injecting drug user; MSM: Men who have sex with men; TRI: Test for recent infection.

\section{Acknowledgements}

This work was supported in part by the Italian Ministry of Health (Fondi Ricerca Corrente INMI "L. Spallanzani", Progetto AIDS grant no. n. 40H78, Progetto Ricerca Finalizzata 107/03).

We thank Margaret Becker for her help in revising the English quality of the manuscript.

Persons and institutions who contributed to the SENDIH study are: INMI L. Spallanzani - Roma: Orchi N, Balzano R, Elia P, Navarra A, Nurra G, Palummieri A, Puro V, De Carli G, Fusco FM, Pittalis S, Scognamiglio P, Ammassari A, Bellagamba R, Boumis E, Corpolongo A, D'Offizi G, Galati V, Grisetti S, Liuzzi G, Nicastri E, Sampaolesi A, Visco Comandini U, Zaccarelli M, Tozzi V, Narciso P, Petrosillo N, Antinori A, Perno CF, D’Arrigo R, Gori C, Capobianchi M, Zaniratti S, Selleri M, Sias C, Sciarrone MR, Ippolito G, Girardi E. Policlinico Umberto I - Roma: Falciano M, Vullo V. Policlinico di Torvergata - Roma: Sarmati ML, Battagin G, Buonomini AR, Andreoni M. Istituto San Gallicano - Roma: Giuliani M, Di Carlo A. U.O. AIDS ASL RMB - Roma: Brancatella R, Maggi T, Errico F. U.O. AIDS ASL RMD - Ostia: Schito S. U.O. AIDS ASL RME - Roma: Gattari P, Spizzichino L. U.O. AIDS ASL RMH - Frascati: Francesconi M. U.O. AIDS Ospedale S. Eugenio - Roma: De Filippis A. CRAIDS Ospedale di Latina - Latina: Soscia F, Roberti L, Tacconi L. CRAIDS Ospedale di Frosinone - Frosinone: Gallo I, Anzalone E. CRAIDS Ospedale di Rieti - Rieti: Natalini G, Raponi A, Pitorri A. CRAIDS Ospedale di Viterbo - Viterbo: Caterini A, S. Aviani Barbacci S.

\section{Author details}

${ }^{1}$ Istituto Nazionale per le Malattie Infettive "Lazzaro Spallanzani", Rome, Italy ${ }^{2}$ Laziosanità-Agenzia di Sanità Pubblica, Rome, Italy.

\section{Authors' contributions}

AM, PP, CA, NO, and EG conceived the initial idea and the study design; AM implemented the model and drafted the manuscript; PP and CA linked the data, contributed to data analysis and results interpretation; AN managed the database of SENDIH study and extracted the data; NO, VP, EG, and GI coordinated the SENDIH study and contributed to data interpretation; PP, AC, GG and PB coordinated the Regional Surveillance System and contributed to data interpretation; MRS and CS were responsible for the laboratory analyses. AM, PP, CA, NO and EG revised the manuscript. All authors read and approved the final manuscript.

\section{Competing interests}

The authors declare that they have no competing interests.

Received: 28 June 2011 Accepted: 20 March 2012

Published: 20 March 2012

\section{References}

1. Busch MP, Pilcher CD, Mastro TD, Kaldor J, Vercauteren G, Rodriguez W, Rousseau C, Rehle TM, Welte A, Averill MD, Garcia Calleja JM: Beyond detuning: 10 years of progress and new challenges in the development and application of assays for HIV incidence estimation. AIDS 2010, 24(18):2763-2771.

2. Barin F, Nardone A: Monitoring HIV epidemiology using assays for recent infection:where are we? Euro Surveill 2008, 13:18967.

3. Janssen RS, Satten GA, Stramer SL, Rawal BD, O'Brien TR, Weiblen BJ, Hetch FM, Jack N, Cleghorn FR, Kahn JO, Chesney MA, Busch MP: New testing strategy to detect early HIV-1 infection for use in incidence 
estimates and for clinical and prevention purposes. JAMA 1998 280:42-48.

4. Dobbs T, Kennedy S, Pau CP, McDougal JS, Parekh BS: Performance characteristics of the immunoglobulin G-capture BED-enzyme immunoassay, an assay to detect recent human immunodeficiency virus type 1 seroconversion. J Clin Microbiol 2004, 42:2623-2628

5. Murphy G, Parry JV: Assays for the detection of recent infections with human immunodeficiency virus type 1. Euro Surveill 2008, 13(36):4-10.

6. Cooper DA, Imrie AA, Penny R: Antibody response to human immunodeficiency virus after primary infection. J Infect Dis 1987, 155:1113-1118.

7. Lange JMA, Parry JV, De Wolf F, Mortimer PP, Goudsmit J: Diagnostic value of specific IgM antibodies in primary HIV infection. AIDS 1988, 2:31-35.

8. Fiebig EW, Wright DJ, Rawal BD, Garrett PE, Schumacher RT, Peddada L, Heldebrant C, Smith R, Conrad A, Kleinman SH, Busch MP: Dynamics of HIV viremia and antibody seroconversion in plasma donors: implications for diagnosis and staging of primary HIV infection. AIDS 2003, 17:1871-1879.

9. Guy R, Gold J, Garcia Calleja JM, Kim AA, Parekh B, Busch M, Rehle T, Hargrove J, Remis RS, Kaldor JM: Accuracy of serological assays for detection of recent infection with HIV and estimation of population incidence: a systematic review. Lancet Infect Dis 2009, 9:747-759.

10. Schwarcz S, Weinstock H, Louie B, Kellogg T, Douglas J, Lalota M, Dickinson G, Torian L, Wendell D, Paul S, Goza G, Ruiz J, Boyett B, McCormick L, Bennett D: Characteristics of persons with recently acquired HIV infection: application of the serologic testing algorithm for recent HIV seroconversion in 10 US cities. J Acquir Immune Defic Syndr 2007, 44(1):112-115.

11. Semaille C, Cazein F, Pillonel J, Lot F, Le Vu S, Pinget R, Desenclos J, Barin F: Four years of surveillance of recent HIV infections at country level, France, mid 2003-2006: experience and perspectives. Euro Surveill 2008, 13(36):17-22.

12. Puchhammer-Stockl E, Schmied B, Rieger A Sarcletti M, Geit M, Zangerle R, Hofmann H: Low proportion of recent Human Immunodeficiency Virus (HIV) infections among newly diagnosed cases of HIV infection as shown by the presence of HIV-specific antibodies of low avidity. J Clinical Microbiol 2005, 43:497-498.

13. Bätzing-Feigenbaum J, Loschen S, Gohlke-Micknis S, Hintsche B, Rausch M, Hillenbrand $H$, Cordes $C$, Poggensee G, Kücherer C, Hamouda O: Implications of and perspectives on HIV surveillance using a serological method to measure recent HIV infections in newly diagnosed individuals: results from a pilot study in Berlin, Germany, in 2005-2007. HIV Med 2009, 10:209-218.

14. Romero A, González V, Granell M, Matas L, Esteve A, Martró E, Rodrigo I, Pumarola T, Miró JM, Casanova A, Ferrer E, Tural C, del Romero J, Rodríguez C, Caballero E, Ribera E, Casabona J, Standardized Algorithm for Recent HIV Infections (AERIVIH) study group: Recently acquired HIV infection in Spain (2003-2005): introduction of the serological testing algorithm for recent HIV seroconversion. Sex Transm Infect 2009, 85(2):106-110.

15. Cortes Martins H, Paixão MT: Settings for identifying recent HIV infections: the Portuguese experience. Euro Surveill 2008, 13(36):1-6.

16. Hall HI, Song R, Rhodes P, Prejean J, Quian A, Lee LM, Karon J, Brookmeyer R, Kaplan EH, McKenna MT, Janssen RS: Estimation of HIV incidence in the United States. JAMA 2008, 300:520-529.

17. Karon JM, Song R, Brookmeyer R, Kaplan EH, Hall IH: Estimating HIV incidence in the United States from HIV/AIDS surveillance data and biomarker HIV test results. Stat Med 2008, 27:4617-4633.

18. Le Vu S, Le Strat Y, Barin F, Pillonel J, Cazein F, Bousquet V, Brunet S, Thierry D, Semaille C, Meyer J, Desenclos JC: Population-based HIV-1 incidence in France, 2003-08: a modelling analysis. Lancet Infect Dis 2010, 10:682-687.

19. Brancato G, Perucci CA, Abeni DD, Sangalli M, Ippolito G, Arcà M: The changing distribution of HIV infection: HIV surveillance in Lazio, Italy, 1985 through 1994. Am J Public Health 1997, 87:1654-1658.

20. Orchi N, Balzano R, Scognamiglio P, Navarra A, De Carli G, Elia P, Grisetti S, Sampaolesi A, Giuliani M, De Filippis A, Puro V, Ippolito G, Girardi E, SENDIH group: Ageing with HIV: newly diagnosed older adults in Italy. AIDS Care 2008, 20:419-425.

21. Suligoi B, Massi M, Galli C, Sciandra M, Di Sora F, Pezzotti P, Recchia O, Montella F, Sinicco A, Rezza G: Identifying recent HIV infections using the avidity index and an automated enzyme immunoassay. I Acquir Immune Defic Syndr 2003, 32:424-428.

22. Suligoi B, Galli C, Massi M, Di Sora F, Sciandra M, Pezzotti P, Recchia O, Montella F, Sinicco A, Rezza G: Precision and Accuracy of a Procedure for Detecting Recent Human Immunodeficiency Virus Infections by Calculating the Antibody Avidity Index by an Automated ImmunoassayBased Method. J Clin Microbiol 2002, 11:4015-4020.

23. Selleri M, Orchi N, Zaniratti MS, Bellagamba R, Corpolongo A, Angeletti C, Ippolito G, Capobianchi MR, Girardi E: Effective highly active antiretroviral therapy in patients with primary HIV-1 infection prevents the evolution of the avidity of HIV-1-specific antibodies. J Acquir Immune Defic Syndr 2007, 46:145-150.

24. Chawla A, Murphy G, Donnelly C, Booth CL, Johnson M, Parry JV, Philips A, Geretti AM: Human immunodeficiency virus (HIV) antibody avidity testing to identify recent infection in newly diagnosed HIV type 1 (HIV1)-seropositive persons infected with diverse HIV-1 subtypes. J Clin Microbiol 2007, 45(2):415-420.

25. Martró E, Suligoi B, González V, Bossi V, Esteve A, Mei J, Ausina V: Comparison of the avidity index method and the serologic testing algorithm for recent human immunodeficiency virus (HIV) seroconversion, two methods using a single serum sample for identification of recent HIV infections. J Clin Microbiol 2005, 43(12):6197-6199.

26. Galli C, Bossi V, Regine V, Rodella A, Manca A, Camoni L, Suligoi B: Accuracy of different thresholds for the anti-HIV avidity index. Microbiologia Medica 2008, 23:59-63.

27. Sweeting MJ, De Angelis D, Parry J, Suligoi B: Estimating the distribution of the window period for recent HIV infections: a comparisons of statistical methods. Stat Med 2010, 30:3194-3202.

28. Longini IM Jr, Clark WS, Gardner LI, Brundage JF: The dynamics of CD4+ Tlymphocyte decline in HIV infected individuals: a Markov modelling approach. J Acquir Immune Defic Syndr 1991, 4:1141-1147.

29. Centers for Disease Control and Prevention: Revised Surveillance Case Definitions for HIV Infection Among Adults, Adolescents, and Children Aged $<18$ Months and for HIV Infection and AIDS Among Children Aged 18 Months to $<13$ Years - United States, 2008. MMWR 2008, 57(No. RR-10):1-12.

30. Carnegie NB: Bootstrap confidence intervals and bias correction in the estimation of HIV incidence from surveillance data with testing for recent infection. Statist Med 2011, 30:854-865.

31. 2010 Annual report on the state of the drugs problem in Europe European Monitoring Centre for Drugs and Drug Addiction, Lisbon; 2010.

32. Immigrazione, Dossier Statistico Caritas/Migrantes, XX Rapporto Idos Edizioni; 2010.

33. Barbagli M, Dalla Zuanna G, Garelli F: La sessualità degli italiani Bologna: II Mulino; 2010.

34. R Development Core Team: R: A Language and Environment for Statistical Computing R Foundation for Statistical Computing; 2010.

35. Su YS, Gelman A, Hill J, Yajima M: Multiple Imputation with Diagnostics (mi) in R: Opening Windows into the Black Box. I of Statistical Software 2011, 45(2):1-31.

36. Downs AM, Heisterkamp SH, Brunet JB, Hamers FF: Reconstruction and Prediction of the HIV/AIDS Epidemic among Adults in the European Union and in the Low Prevalence Countries of Central and Eastern Europe. AIDS 1997, 11:649-662.

37. Barcherini S, Cantoni M, Grossi P, Verdecchia A: Reconstruction of Human Immunodeficiency Virus (HIV) Sub-epidemics in Italian Regions. Int $J$ Epidemiol 1999, 28(1):122-129.

38. Bellocco R, Pagano M: Multinomial Analysis of Smoothed HIV Backcalculation Models Incorporating Uncertainty in the AIDS Incidence. Statist Med 2001, 20:2017-2033.

39. Suligoi B, Boros S, Camoni L, Lepore D, Occhiodoro V: Aggiornamento delle nuove diagnosi di infezione da HIV al 31 dicembre 2008 e dei casi di AIDS in Italia al 31 dicembre 2009. Not Ist Super Sanità 2010, 23(4 Suppl):3-27.

40. Prejean J, Song R, Hernandez A, Ziebell R, Green T, Walker F, Lin LS, An Q, Mermin J, Lansky A, Hall IH, for the HIV Incidence Surveillance Group: Estimated HIV Incidence in the United States, 2006-2009. PLoS One 2011, 6(8):e17502, Epub 2011 Aug 3. 
41. Jenness SM, Neaigus A, Murrill CS, Wendel T, Forgione L, Hagan H: Estimated HIV Incidence among High-risk Heterosexuals in New York City, 2007. J Acquir Immune Defic Syndr 2011, 56(2):193-197.

42. MacKellar DA, Valleroy LA, Secura GM, Bartholow BN, McFarland W, Shehan D, Ford W, LaLota M, Celentano DD, Koblin BA, Torian LV, Perdue TE, Janssen RS: Repeat HIV testing, risk behaviors, and HIV seroconversion among young men who have sex with men: a call to monitor and improve the practice of prevention. J Acquir Immune Defic Syndr 2002, 29(1):76-85.

43. Kellerman SE, Lehman JS, Lansky A, Stevens MR, Hecht FM, Bindman AB, Wortley PM: HIV testing within at-risk populations in the United States and the reasons for seeking or avoiding HIV testing. I Acquir Immune Defic Syndr 2002, 31(2):202-210.

44. Nwanyanwu OC, Conti LA, Ciesielski CA, Stehr-Green JK, Berkelman RL, Lieb S, Witte JJ: Increasing frequency of heterosexually transmitted AIDS in southern Florida: artifact or reality? Am J Public Health 1993, 83(4):571-573.

Pre-publication history

The pre-publication history for this paper can be accessed here: http://www.biomedcentral.com/1471-2334/12/65/prepub

doi:10.1186/1471-2334-12-65

Cite this article as: Mammone et al:: HIV incidence estimate combining HIV/AIDS surveillance, testing history information and HIV test to identify recent infections in Lazio, Italy. BMC Infectious Diseases 2012 12:65.

\section{Submit your next manuscript to BioMed Central} and take full advantage of:

- Convenient online submission

- Thorough peer review

- No space constraints or color figure charges

- Immediate publication on acceptance

- Inclusion in PubMed, CAS, Scopus and Google Scholar

- Research which is freely available for redistribution

Submit your manuscript at www.biomedcentral.com/submit 